[1]

'Dept of Respiratory Medicine, A.J. Institute of Medical Sciences, Mangalore, India

${ }^{2}$ Light House Polyclinic, Mangalore, India

\title{
A rare cause for a common symptom
}

\section{Case report}

Cite as: Sharma MV, Kakkilaya BS, Shekh IA, et al. A rare cause for a common symptom.

Breathe 2016; 12: e64-e74.
A 56-year-old male patient, Hindu priest by profession for the last 32 years, presented with a history of dry cough and evening rise of temperature for the past 6 months. He had no other respiratory symptoms or any symptoms referable to other systems. He had no weight loss or anorexia. He was a diabetic and had been taking metformin for the past 10 years. He was also on amlodipine $5 \mathrm{mg}$ once daily for the past 8 years, for systemic hypertension.

\section{Task 1}

Which of the following is least likely to be the cause for his symptoms?
a) Pulmonary tuberculosis
b) Upper airway cough syndrome
c) Interstitial lung disease (ILD)
d) Chronic hypersensitivity pneumonitis
e) Tropical pulmonary eosinophilia 


\section{Answer 1}

c) ILD

The patient had no breathlessness, so ILD is least likely. Fever and chronic dry cough can occur in all the other conditions.

\section{Task 2}

Which of the following conditions is least likely to cause chronic cough with fever?

a) Chronic liver abscess/subphrenic abscess

b) Large bowel malignancy

c) Autoimmune/connective tissue diseases

d) Haematopoietic and lymphoid malignancies

e) Tuberculous pericarditis

Sometimes nonrespiratory conditions can also lead to chronic cough with fever.

\section{Answer 2}

b) Large bowel malignancy

Large bowel malignancy is least likely to cause chronic cough with fever. Subdiaphragmatic lesions like liver abscess/subphrenic abscess can irritate the diaphragm to cause chronic cough. Some cough receptors are also present in the pericardium; hence, tuberculous pericarditis can lead to chronic cough with fever. Haematopoietic and lymphoid malignancies can also lead to chronic cough with fever as they are more prone to respiratory infection, sometimes due to direct lung parenchymal involvement.

Some drugs can also lead to chronic cough with fever. Therefore, medication history is very important.

\section{Task 3}

Which of following groups of drugs is least likely to cause chronic cough?
a) Tricyclic antidepressants
b) Angiotensin-converting enzyme (ACE) inhibitors
c) Beta blockers
d) Nonsteroidal anti-inflammatory drugs (NSAIDs)
e) Calcium channel blockers and nitrates 


\section{Answer 3}

a) Tricyclic antidepressants

Beta blockers can cause cough by inducing bronchospasm in some individuals with bronchial asthma. NSAIDs can lead to cough in atopic individuals and are usually associated with nasal symptoms. Calcium channel blockers and nitrates are also known to cause chronic cough. ACE inhibitors cause dry nocturnal cough in about $5-35 \%$ of users. Inhibition of bradykinin metabolism results in ACE-induced cough.

Apart from in the airways, cough receptors are present in many other sites. Any lesion in these areas also can produce cough.

\section{Task 4}

Which one of the following sites does not have cough receptors?
a) Diaphragm
b) Pericardium
c) Oesophagus
d) Stomach
e) Peritoneum

\section{Answer 4}

e) Peritoneum

Apart from in the major airways, cough receptors are present in the upper airways, external and internal ear, pleura, diaphragm, pericardium, oesophagus and stomach. Hence, any disease involving these organs can also manifest with cough.

\section{Further history}

The patient had no upper airway symptoms, no upper gastrointestinal symptoms and no history of breathlessness. There was no change in symptoms during his work. He had no cardiac symptoms and no symptoms suggestive of autoimmune/ connective tissue disease. He was not on any medication that could cause cough. There was no history of any psychological illness. He had consulted a family physician and was treated with antipyretic and antitussive medications, but there was no relief to his symptoms.

\section{Physical findings}

General examination, respiratory system examination and other systemic examinations were unremarkable. Chest radiography was normal (figure 1).

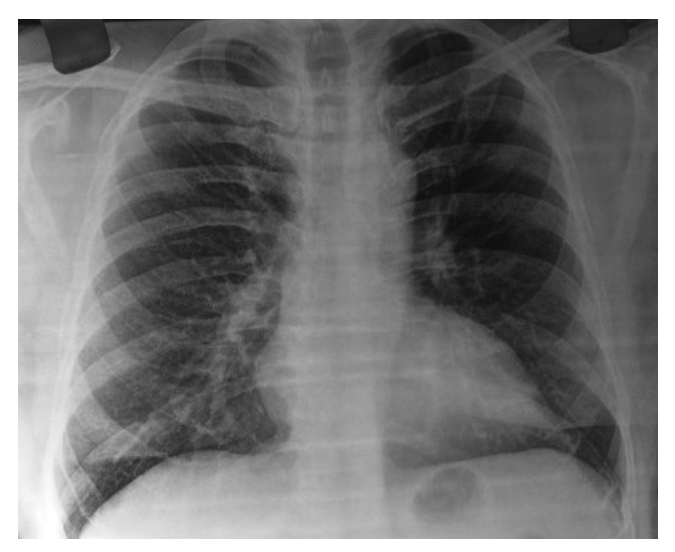

Figure 1 Normal chest radiograph.

\section{Task 5}

Which of the following is least likely to cause chronic dry cough without any other obvious symptoms or signs?
a) Hyperreactive airway disease
b) Chronic obstructive pulmonary disease (COPD)
c) Gastro-oesophageal reflux disease (GORD)
d) Small intrathoracic tumours
e) Endobronchial lesion 


\section{Answer 5}

\section{b) COPD}

In patients with COPD, breathlessness is the predominant symptom besides chronic cough. Some patients with GORD may not have gastrointestinal symptoms and may present with chronic cough more at night.

\section{Investigations}

Investigations were performed and the results are shown in table 1.

\section{Task 6}

What is the next investigation?
a) Ultrasound of the abdomen
b) Bronchoscopy
c) Computed tomography (CT) scan of thorax
d) Spirometry
e) Tumour markers

Table 1 Investigation results

\begin{tabular}{|c|c|}
\hline Parameter & Result \\
\hline Haemoglobin $\mathrm{g} \cdot \mathrm{dL}^{-1}$ & 13.2 \\
\hline Total leukocyte count cells $\cdot \mathrm{mm}^{-3}$ & 6400 \\
\hline \multicolumn{2}{|l|}{ Differential leukocyte count \% } \\
\hline Neutrophils & 59 \\
\hline Lymphocytes & 39 \\
\hline Eosinophils & 2 \\
\hline Monocytes & 0 \\
\hline ESR $\mathbf{m m} \cdot \mathbf{h}^{-1}$ & 112 \\
\hline Liver and renal function tests & Normal \\
\hline FBS $\mathrm{mg}^{\mathrm{d}} \mathrm{dL}^{-1}$ & 159 \\
\hline PPBS $m g \cdot d L^{-1}$ & 200 \\
\hline Urine routine examination & No abnormality detected \\
\hline Haemoglobin A1c \% & 7.3 \\
\hline Peripheral smear examination for malaria parasite & Negative \\
\hline Serology (HIV, HCV, HBsAg) & Negative \\
\hline
\end{tabular}

ESR: erythrocyte sedimentation rate; FBS: fasting blood sugar; PPBS: post-prandial blood sugar; $\mathrm{HCV}$ : hepatitis C virus; $\mathrm{HBsAg}$ : hepatitis B surface antigen. 


\section{Answer 6}

a) Ultrasound of the abdomen

Ultrasound of the abdomen is the next simplest investigation to check for any infradiaphragmatic cause. If ultrasound is normal, further evaluation can be performed. Spirometry and tumour markers are not preferred as the initial evaluation in chronic cough with fever.

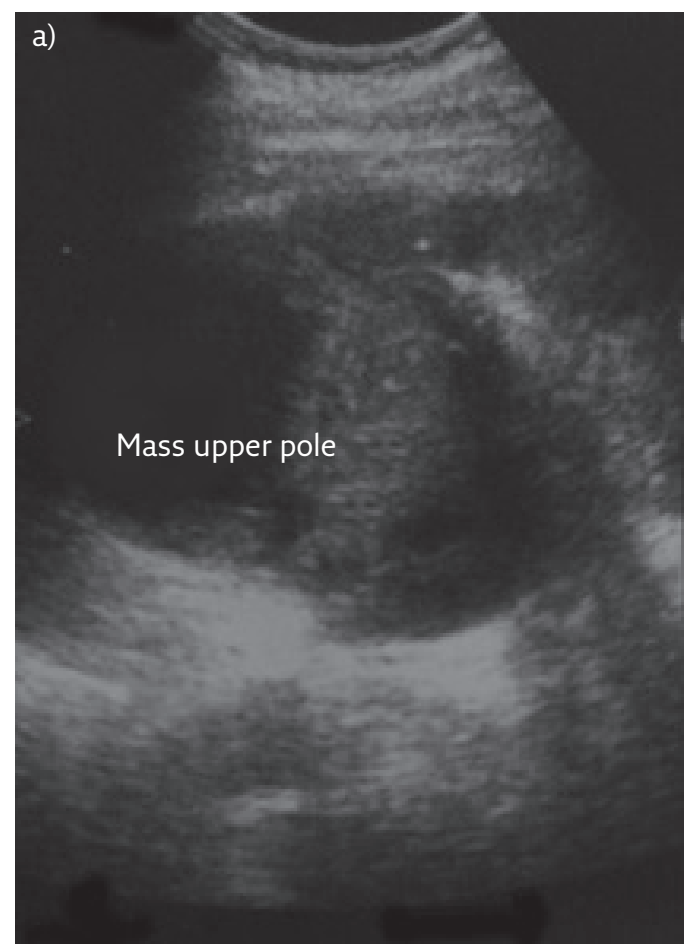

Ultrasound showed a left renal mass with necrosis, $10 \times 8 \mathrm{~cm}$ in size, in the upper pole (figure 2). The ultrasound findings were suggestive of renal cell carcinoma (RCC).

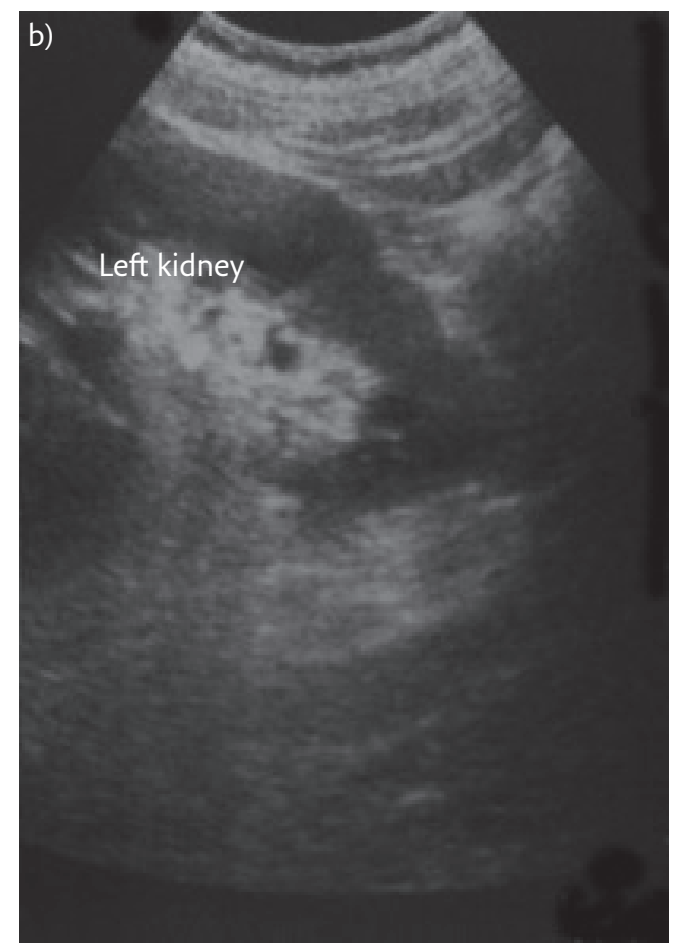

Figure 2 Ultrasound of the abdomen. a) Oblique axial image of the left kidney, showing an ill-defined heterogeneous hypoechogenic mass lesion, possibly arising from the upper and interpolar regions of the left kidney. b) Long-axis sagittal view of the left kidney, confirming the mass is located in the upper pole.

\section{Task 7}

What is the next investigation?
a) Laparoscopy and biopsy
b) Contrast-enhanced CT (CECT) scan of abdomen and chest
c) Tumour markers
d) Laparotomy
e) Percutaneous biopsy 


\section{Answer 7}

\section{b) CECT}

The preferred investigation in suspected RCC is $\mathrm{CECT}$, as $\mathrm{CT}$ will show the extent of the tumour and enable planning for further treatment.
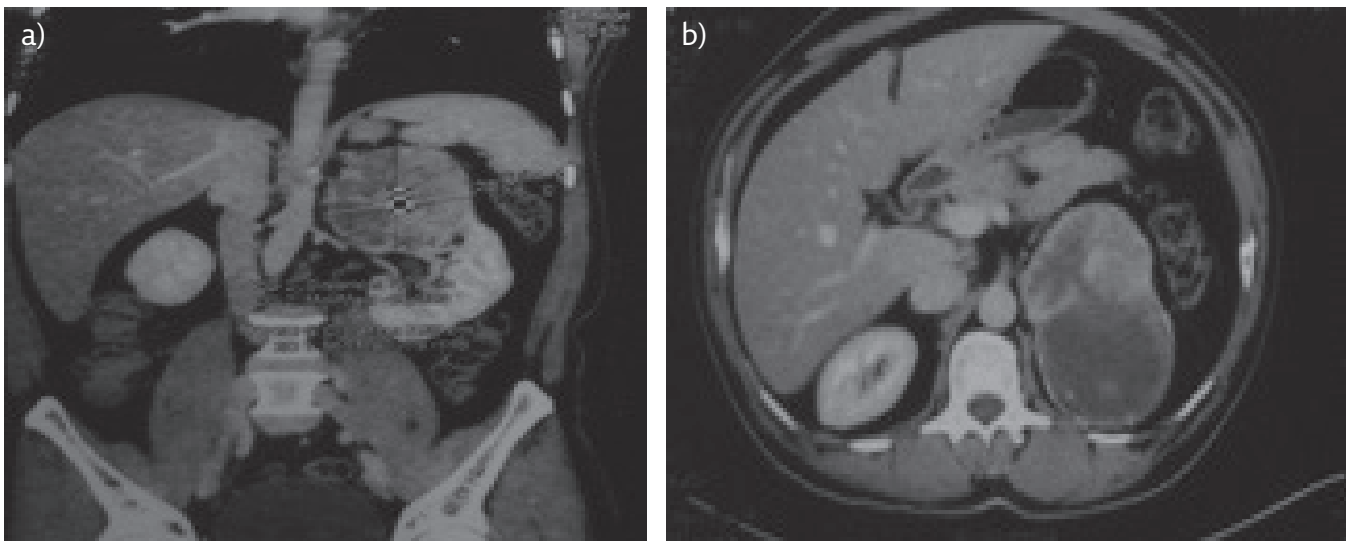

Figure 3 CECT of the abdomen, showing a) coronal view, arterial phase, and b) axial view at the upper lumbar level, T12 to L1 vertebral level.
The CECT showed a heterogeneously enhancing well-encapsulated mass lesion arising from the upper pole of the left kidney, suggestive of RCC (figure 3). There was no evidence of metastasis or invasion into the surrounding structures.

\section{Task 8}

What is the most common cause for cough in RCC?
a) Secondaries in lung parenchyma
b) Large tumour irritating diaphragm
c) Lymphangitis carcinomatosis
d) Endobronchial secondaries
e) Malignant pleural effusion 


\section{Answer 8}

a) Secondaries in lung parenchyma

The most common site for secondaries in RCC is the lungs. This can manifest with chronic cough.

CECT did not reveal any parenchymal lesion or mediastinal lympadenopathy. Other intrathoracic structures were normal (figure 4).

\section{Task 9}

What could be the cause for chronic cough?
a) Endobronchial secondaries
b) Small parenchymal secondaries
c) Pleural effusion
d) Tumour emboli
e) Lymphangitis carcinomatosis
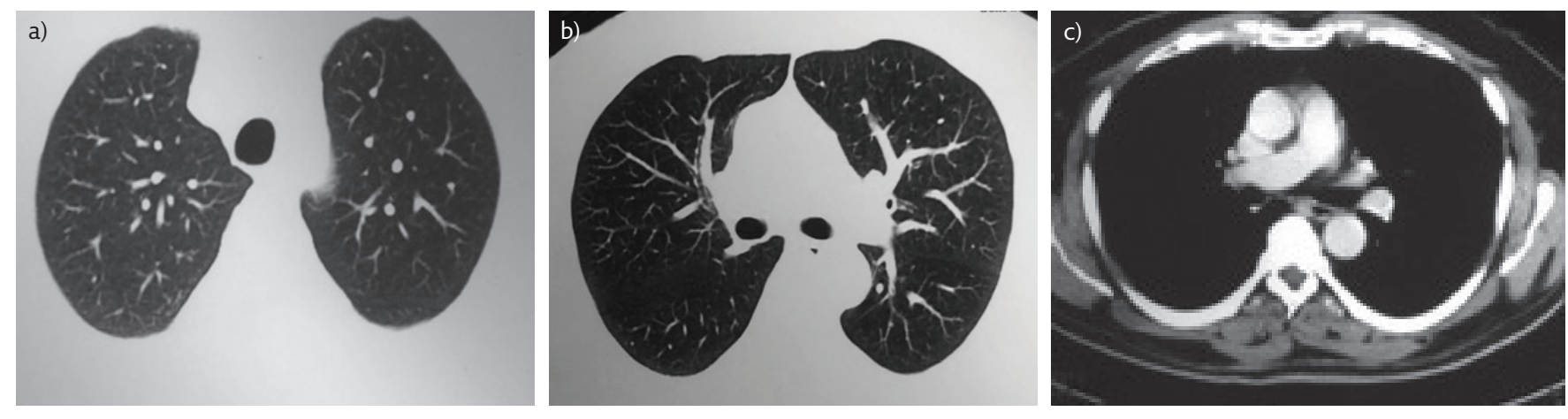

Figure 4 CT of the thorax, showing a) lungs at T3 vertebral level, b) lungs at $T 7$ vertebral level, and c) post-contrast mediastinal view at $\Pi 7$ vertebral level. 


\section{Answer 9}

a) Endobronchial secondaries

Endobronchial secondaries sometimes may not be visible by CT. In tumour emboli and lymphangitis carcinomatosis, the predominant symptom would be breathlessness, which this patient did not have.

\section{Task 10}

Which is the most common primary site in endobronchial secondaries?
a) Malignant melanoma
b) Kidney
c) Colon
d) Breast
e) Sarcoma

\section{Task 11}

What is the next investigation? 


\section{Answer 10}

b) Kidney

\section{Answer 11}

Bronchoscopy

Bronchoscopy was normal.

\section{Task 12}

What could be the cause for fever and cough?
a) Paraneoplastic manifestation of RCC
b) Occult secondary
c) Tumour emboli
d) Diaphragmatic irritation by the tumour
e) Pericardial effusion 


\section{Answer 12}

a) Paraneoplastic manifestation of RCC

RCC can present with chronic cough and fever as paraneoplastic manifestations. There was no evidence of pericardial effusion or extension, or involvement of the diaphragm, on the CT scan.

\section{Treatment}

As the tumour was confined, the patient was treated with radical nephrectomy. The cough subsided within 4 days of surgery. The gross specimen is shown in figure 5 and histopathology showed clear cell carcinoma (figure 6).

\section{Discussion}

\section{Mechanism of chronic cough and fever in RCC}

RCC secretes prostaglandins (mainly prostaglandin E2) and cytokines that have been implicated in enhancing the cough reflex via the EP3 receptor pathway. The constitutional symptoms found in RCC are thought to be mediated by cytokines. Patients with RCC are known to have elevated serum levels of tumour necrosis factor (TNF)- $\alpha$ and interleukin- 6 , which are pyrogens leading to fever in RCC. TNF- $\alpha$ is a cytokine known to alter adipocyte metabolism and affect appetite control.

$10-40 \%$ of patients with RCC develop paraneoplastic manifestations. In up to one third of cases, symptoms such as fever, weight loss and fatigue are the first symptoms of RCC. Fever is found in $20-30 \%$ of those with RCC and is the sole presenting complaint in approximately $2 \%$ of patients. The constitutional symptoms found in RCC are thought to be mediated by cytokines.

\section{Learning points}

Chronic dry cough and fever can occur in a variety of infective, neoplastic, autoimmune and connective tissue disorders. Fever and cough can occur even in extrathoracic diseases. Paraneoplastic manifestation of RCC is a rare cause for chronic dry cough with fever.
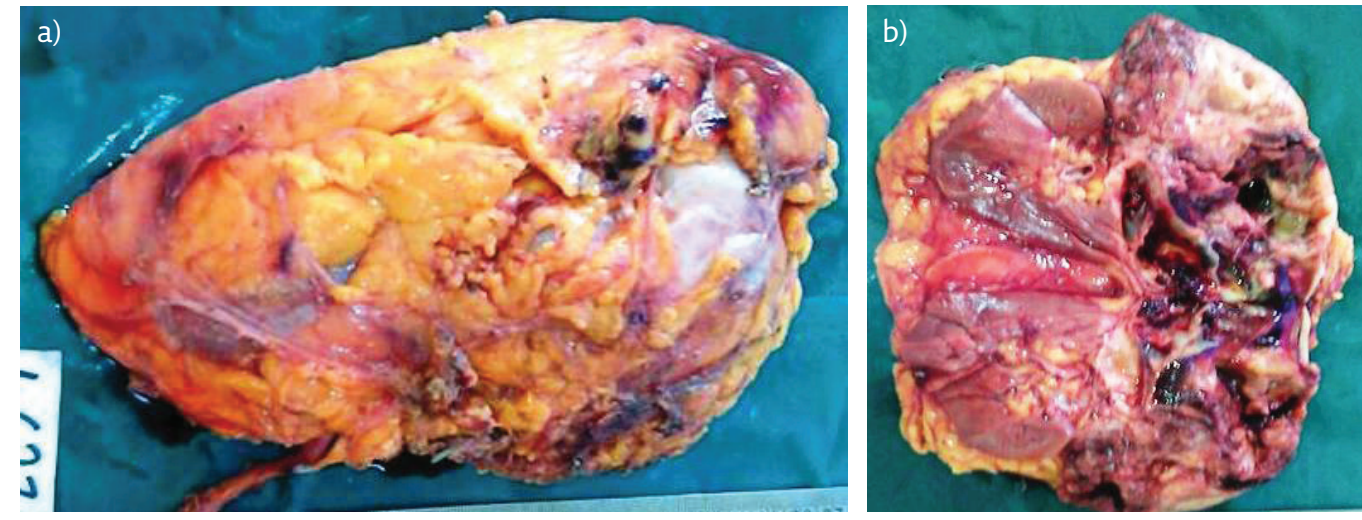

Figure 5 Gross specimen, showing a) the outer surface of the nephrectomy specimen with intact perinephric fat, and b) the cut surface of the left kidney, which shows an ill-defined tumour mass with a variegated appearance situated in the upper pole.
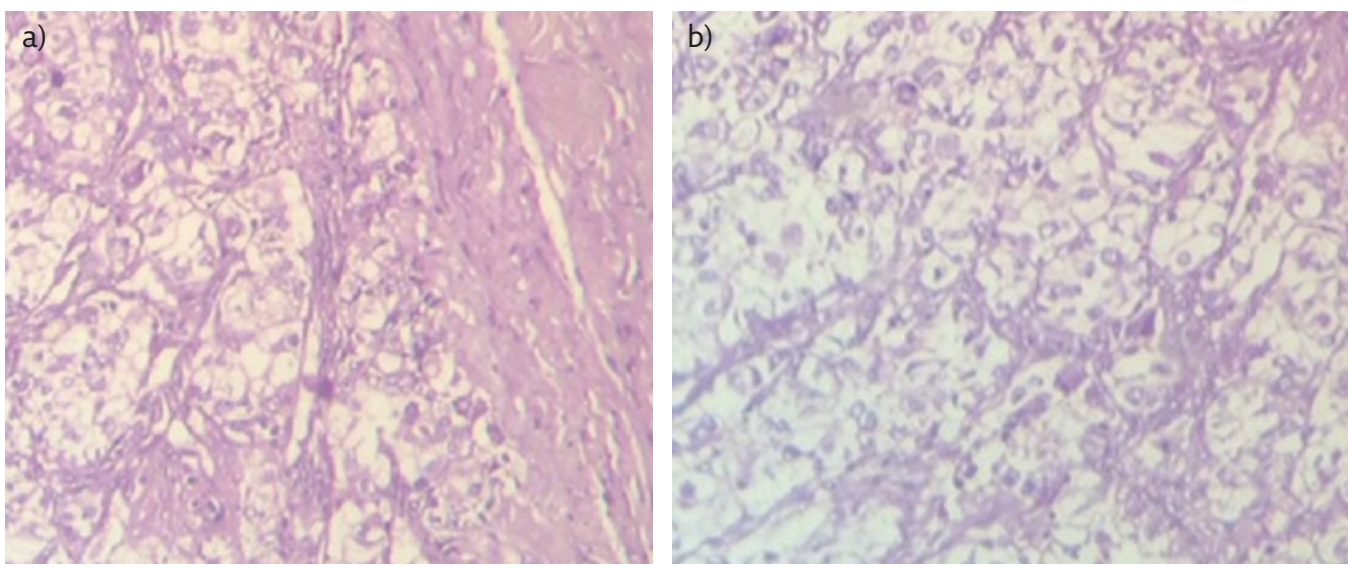

Figure 6 Haematoxylin and eosin stain at a) 100x and b) 400x magnification. Tumour cells are arranged in sheets and lobular pattern, with malignant clear cells diagnostic of RCC. 


\section{Conflict of interest}

None declared.

\section{Further reading}

- Okubo Y, Yonese J, Kawakami S, et al. Obstinate cough as a sole presenting symptom of non-metastatic renal cell carcinoma. Int J Urol 2007; 14: 854-855.

- Palapattu GS, Kristo B, Rajfer J. Paraneoplastic syndromes in urologic malignancy: the many faces of renal cell carcinoma. Rev Urol 2002; 4: 163-170.
- Chandrankunnel J, Cunha BA, Petelin A, et al. Fever of unknown origin (FUO) and a renal mass: renal cell carcinoma, renal tuberculosis, renal malakoplakia, or xanthogranulomatous pyelonephritis? Heart Lung 2012; 41: 606-609.

- Tatzel S, Sener A. Persistent dry cough: an unusual presentation of renal cell carcinoma. CMAJ 2014; 186: 136. 